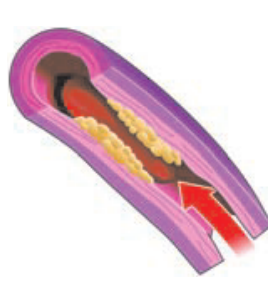

\title{
The role of statin therapy in patients with lower vascular risk
}

\author{
New evidence from an international prospective meta-analysis should be \\ incorporated into clinical and Pharmaceutical Benefits Scheme guidelines
}

\section{Anthony C Keech MB BS, MSC, FRACP Deputy Director \\ Jordan R Fulcher BSc(Med), MB BS, Research Fellow \\ John Simes MD, SM, FRACP, Director \\ NHMRC Clinical Trials Centre, University of Sydney, Sydney, NSW. \\ tony@ctc.usyd.edu.au}

doi: 10.5694/mjal2.10925

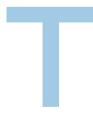
here is no doubting the fundamental role of statin therapy in patients with established cardiovascular disease. However, its role in primary prevention, particularly among patients with lower absolute cardiovascular risk, has been uncertain. ${ }^{1}$ In 2010 , in a meta-analysis of data from 170000 individual patients with or without cardiovascular disease, the Cholesterol Treatment Trialists' Collaboration (CTTC) showed that each $1 \mathrm{mmol} / \mathrm{L}$ reduction in low-density lipoprotein (LDL)-cholesterol level was associated with a reduction in the incidence of major vascular events of about $20 \%$ over 5 years, even among those with starting LDL-cholesterol levels less than $2.0 \mathrm{mmol} / \mathrm{L}^{2}{ }^{2}$ But is this benefit equally applicable to subgroups of patients who have no history of cardiovascular disease and those who have a lower absolute cardiovascular risk? As at least half of all vascular events occur among the latter patient group, ${ }^{3}$ resolving this uncertainty is important.

Recently, the CTTC conducted a further meta-analysis according to risk of vascular disease, ${ }^{3}$ using their protocol for meta-analysis that prospectively describes study entry criteria, analytical methods and common end points, and thus has greater validity than a protocol formulated posthoc. The CTTC also has access to patient-level data, which provides more reliable estimates of treatment effects than a standard meta-analysis. Patients were stratified at baseline into five arbitrary whole-number categories of 5-year risk of a major vascular event: $<5 \% ; 5 \%$ to $<10 \% ; 10 \%$ to $<20 \%$; $20 \%$ to $<30 \%$; or $\geqslant 30 \%$. The reduction in major vascular events among each group was similar to the overall reduction of $21 \%$ for each $1 \mathrm{mmol} / \mathrm{L}$ reduction in LDL-cholesterol level.

Among patients with no history of vascular disease, those in the two lowest-risk groups who received treatment had significant proportional reductions in the risk of a major vascular event, which were at least as large as reductions among other patients in higher-risk groups. Cardiovascular mortality was reduced by 15\% (and overall mortality by $9 \%$ ), even when patients with diabetes or chronic kidney disease were excluded from the analysis. Non-vascular mortality, cancer incidence and cancer mortality were not affected.

Among participants with a 5-year risk of less than 10\%, around 11 major vascular events were avoided per 1000 treated over 5 years for each $1 \mathrm{mmol} / \mathrm{L}$ reduction in LDLcholesterol level (4.1\% incidence in statin or higher-dose therapy group versus $5.2 \%$ in the control or low-dose statin therapy group).

Over 5 years of treatment, 1000 patients have about 1.2 adverse events, including higher incidences of myopathy (about 0.5 per 1000) and rhabdomyolysis (about 0.1 per 1000); ${ }^{4}$ a small increased risk of developing diabetes, resulting in up to 0.2 additional cardiovascular events per $1000,{ }^{3}$ and the possibility of a higher incidence of haemorrhagic stroke (about 0.5 per 1000). ${ }^{2}$ One potential adverse effect has been shown by postmarketing surveillance reports - a possible rare association of statin treatment with mild cognitive impairment that appears to be reversible over a median time of 3 weeks after treatment withdrawal. ${ }^{5}$ No adverse link between statin treatment and dementia has been found, and reassuringly, the Heart Protection Study found no differences in cognitive function, dementia or other psychiatric disorders after 5 years of statin therapy or placebo among 20000 people. $^{6}$

Australian guidelines published in 2012 by the National Vascular Disease Prevention Alliance and endorsed by the National Health and Medical Research Council encourage use of a 5-year absolute cardiovascular risk calculation (derived from the Framingham risk score) in determining the role for lipid-lowering and other cardiovascular risk therapy. ${ }^{7}$ In primary prevention, lifestyle modification is the empirical recommendation for those with a 5-year cardiovascular risk of less than $10 \%$. Among those whose risk is $10 \%-15 \%$, lipid-lowering therapy should be considered when lifestyle modification has been unsuccessful or risk factors are persistent (eg, hypertension) or unmodifiable (eg, ethnicity or family history). Among those with a 5year risk greater than $15 \%$, lipid-lowering therapy is recommended along with lifestyle modification. The National Institute of Clinical Excellence guidelines do not recommend statin therapy until 5-year risk exceeds $10 \%{ }^{8}$

The CTTC analysis has shown that lowering LDL levels with statin therapy is effective in reducing cardiovascular events even among patients with relatively low absolute risk, and suggests a likely net benefit in favour of statin therapy in this population. In light of this new evidence, should the indications for lipid-lowering therapy be broadened? 
First, the population with lower absolute risk will generally derive a lower net benefit from statin therapy. Second, while some adverse events, such as new diabetes and rhabdomyolysis, appear to be at least partly dose related, other events may not be, and must be factored into clinical decision making. Third, the strength of evidence of net clinical benefit is not as great in the lower-risk groups as among those at higher risk. This is because current confidence intervals around evidence for absence of harm still leave room (at least theoretically) for slight adverse effects from cancer and non-cardiovascular mortality. This therefore could potentially offset the benefits among those at lower risk. Fourth, for some patients, the inconvenience of medication or the psychological impact of having longterm treatment may be an issue; and fifth, the benefit-torisk ratio is not as reliably known beyond 5-6 years of treatment or for older people. Taking all of this into account, the safest first-line therapy to lower LDL cholesterol is still lifestyle modification, including dietary improvement, exercise and weight loss where applicable. However, risk-to-benefit data indicate a role for statin therapy as well, particularly if conservative measures are insufficient.

The other major consideration is financial. At the end of the 2010-11 financial year, atorvastatin and rosuvastatin comprised two of the top three most dispensed Pharmaceutical Benefits Scheme (PBS) medications, and lipidmodifying therapy was the most costly medication class to the PBS. ${ }^{9}$ PBS guidelines still restrict statin prescribing according to LDL-cholesterol levels stratified by other cardiac risk factors, excluding some individuals whose absolute risk may warrant statin therapy according to the 2012 Australian guidelines. Subsidising treatment for more people on the basis of the new CTTC evidence has financial implications.

Australia has higher generic statin prices than England, and a higher, and increasing, market share of patented statins. ${ }^{10}$ These two factors are argued to have cost the Australian Government up to a billion dollars more during 2005-2009, with cost discrepancies between Australia and England projected to continue. The differences partly relate to restrictions imposed on the choice of subsidised medications in England. Recently in Australia, atorvastatin has come off patent, and rosuvastatin has been reclassified $^{11}$ — steps that should reduce expenditure on statin therapy, although this is likely to be by much less than it could be, based on the experiences of other nations. ${ }^{12}$ These domestic financial factors are important considerations when reviewing the role and capacity for broadened taxpayer-funded subsidy of statins. The Pharmaceutical Benefits Advisory Committee reviewed statin pricing in July 2012, and their decision will be available late in August. Statin prescriptions also incur copayment costs for the individual, but other techniques to lower cholesterol, such as gymnasium use and dietary programs, can also be expensive. In the United Kingdom, low-dose simvastatin has been available over the counter without prescription since $2004 .^{13}$ This strategy may reduce the expense of consultations, although there are potential risks to patients in excluding doctors from the prescription process.

In summary, we now have considerable evidence that lipid-lowering statin therapy reduces cardiovascular events across all examined categories of absolute cardiovascular risk. The side-effect profile of statins is reassuring, even for use in individuals with a 5-year risk of less than $10 \%$, although the benefit-to-harm ratio is greater among those at higher absolute risk. What is still not certain is at what level, clinically or financially, the absolute benefit-to-harm ratio is too low to endorse statin therapy as a routine subsidised treatment. While there is no substitute for lifestyle modification, the capacity for statin pharmacotherapy to assist in the treatment of individuals at lower risk has now been shown. This new evidence must be urgently considered, with appropriate economic analyses, for incorporation into clinical and PBS guidelines.

Acknowledgements: We thank Rhana Pike for assistance with the manuscript.

Competing interests: None of the authors have received industry funding for statin trials during the past 5 years. Anthony Keech and John Simes have received funding for other research; Anthony Keech has received reimbursement for lectures from the pharmaceutical industry, including from companies that market statins.

Provenance: Commissioned; externally peer reviewed.

1 Taylor F, Ward K, Moore TH, et al. Statins for the primary prevention of cardiovascular disease. Cochrane Database Syst Rev 2011; (1): CD004816.

2 Cholesterol Treatment Trialists' (CTT) Collaboration. Efficacy and safety of more intensive lowering of LDL cholesterol: a meta-analysis of data from 170,000 participants in 26 randomised trials. Lancet 2010; 376: 1670-1681.

3 Cholesterol Treatment Trialists' (CTT) Collaborators. The effects of lowering LDL cholesterol with statin therapy in people at low risk of vascular disease: meta-analysis of individual data from 27 randomised trials. Lancet 2012; May 16. [Epub ahead of print.] doi: 10.1016/S0140-6736(12)60367-5.

4 Armitage J. The safety of statins in clinical practice. Lancet 2007; 370: 1781-1790.

5 US Food and Drug Administration. FDA drug safety communication: important safety label changes to cholesterol-lowering statin drugs. Silver Spring, MD: USFDA, 2012. http://www.fda.gov/Drugs/DrugSafety/ucm293101.htm (accessed Jul 2012).

6 Heart Protection Study Collaborative Group. MRC/BHF Heart Protection Study of cholesterol lowering with simvastatin in 20,536 high-risk individuals: a randomised placebo-controlled trial. Lancet 2002; 360: 7-22.

7 National Vascular Disease Prevention Alliance. Guidelines for the management of absolute cardiovascular disease risk. Canberra: National Health and Medical Research Council, 2012. http://www.clinicalguidelines.gov.au/ browse.php?treePath=\&pageType=2\&fldg|r|D=2079\& (accessed Jul 2012).

8 Cooper A, O'Flynn N; Guideline Development Group. Risk assessment and lipid modification for primary and secondary prevention of cardiovascular disease: summary of NICE guidance. BMJ 2008; 336: 1246-1248.

9 Pharmaceutical Benefits Scheme. Expenditure and prescriptions twelve months to 30 June 2011. Canberra: Australian Government Department of Health and Ageing, 2011. http://www.health.gov.au/internet/main/ publishing.nsf/Content/pbs-stats-pbexp-junll (accessed Jul 2012).

10 Clarke PM, Fitzgerald EM. Expiry of patent protection on statins: effects on pharmaceutical expenditure in Australia. Med J Aust 2010; 192: 633-636.

11 Australian Government Department of Health and Ageing. Formulary allocations - 1 May 2012. Canberra: Australian Government DoHA, 2012. http://www.pbs.gov.au/info/industry/pricing/pbs-items/formularyallocations (accessed Jun 2012).

12 Clarke PM. Challenges and opportunities for the Pharmaceutical Benefits Scheme. Med J Aust 2012; 196: 153-154.

13 Sheridan C. Merck's statin first to receive over-the-counter status. Nat Rev Drug Discov 2004; 3: 542-543. 